\title{
Importance of tread inertia and damping on the tyre/road contact stiffness
}

\author{
J. Winroth ${ }^{\mathrm{a}, *}$, P.B.U. Andersson ${ }^{\mathrm{a}}$, W. Kropp ${ }^{\mathrm{a}}$ \\ ${ }^{a}$ Division of Applied Acoustics, Chalmers University of Technology, SE-412 96 Göteborg, \\ Sweden
}

\begin{abstract}
Predicting tyre/road interaction processes like roughness excitation, stickslip, stick-snap, wear and traction requires detailed information about the road surface, the tyre dynamics and the local deformation of the tread at the interface. Aspects of inertia and damping when the tread is locally deformed are often neglected in many existing tyre/road interaction models. The objective of this paper is to study how the dynamic features of the tread affect contact forces and contact stiffness during local deformation. This is done by simulating the detailed contact between an elastic layer and a rough road surface using a previously developed numerical time domain contact model. Road roughness on length scales smaller than the discretisation scale is included by the addition of non-linear contact springs between each pair of contact elements. The dynamic case, with an elastic layer impulse response extending in time, is compared with the case where the corresponding quasistatic response is used. Results highlight the difficulty of estimating a constant contact stiffness as it increases during the indentation process between the elastic layer and a rough road surface. The stiffness-indentation relation additionally depends on how rapidly the contact develops; a faster process gives a stiffer contact. Material properties like loss factor and density also alter the contact development. This work implies that dynamic properties of the local tread deformation may be of importance when simulating contact details during normal tyre/road interaction conditions. There are however indications that the significant effect of damping could approximately be included as an increased stiffness in a quasi-static tread model.
\end{abstract}

\footnotetext{
*Corresponding author. Tel.: +46 31772 2192; fax: +46 317722212.

Email address: julia.winroth@chalmers.se (J. Winroth)
} 
Keywords: tyre/road contact, tyre/road noise, tread modelling

\section{Introduction}

Numerical contact models are essential when optimising the complex tyre/road interaction process. This interaction has a wide range of implications, from rolling resistance and fuel consumption to traction and safety, wear of tyres and roads, and the generation of noise. Fundamentally such contact model includes the two interacting bodies, the road surface and the tyre tread, but the required level of detail and which aspects it must include is often ambiguous.

The tread cap is the outermost layer of the tyre structure providing comfort, traction, and resistance against wear. Standard tread material consists mainly of synthetic and/or natural rubber compounds forming a relatively soft, homogeneous, isotropic and almost incompressible material. Oils, vulcanising chemicals, and fillers such as carbon black and silica are typical substances added to change the material characteristics. Vulcanisation increases the shear modulus by increasing the number of cross-links between polymer chains and polymer chains and fillers. Fillers are used to further change properties, e.g. increasing the modulus while reducing the loss factor. This gives a less pronounced frequency dependence of the modulus compared with uncrossed-linked rubbers (e.g. natural rubber) that has clear terminal, plateau, transition and glassy zones. Generally, tread rubbers show stiffness and relatively large viscous losses that, within the normal working temperatures and frequency range of interest, slowly increase with frequency. More on tread materials can be found in e.g. [1].

In order to simulate tyre/road interaction there are two tread-related mechanisms that are important to consider. Firstly, how the tread influences the dynamic properties of the complete tyre. Secondly and what this paper is concerned with, how the local deformations of the tread in the contact zone affect the contact forces and contact geometry.

Few investigations in the literature seem to be directly focused on the local deformation of the tyre tread. Kropp [2] made one of the first investigations on local deformation by measuring driving point mobilities using different excitation areas. Andersson et al. [3] experimentally and numerically studied the response of the tyre surface for different idealised tread patterns and excitation areas. It was observed that the local deformation 
can be of importance already in the lower frequencies for very small excitation areas. Numerical calculations were done with the model of two elastic layers developed by Larsson and Kropp [4]. This is one of the few tyre models that has also been demonstrated to capture the high-frequency (up to 4 $\mathrm{kHz}$ ) response of the tread and in [3] it was shown that the model accurately captures the local deformation and handles excitation areas down to a few square millimetres. However, the model uses a plate geometry for the tyre structure and it is not able to provide the correct mode-shapes.

An inherent property of tyre/road interaction models is the spatial discretisation of the contact. Contact stiffness within an element, connected to the roughness profile within the element boundaries, can be assumed either infinite (Lagrange multipliers/no penetration condition), constant (linear contact springs) or non-linear. The contact mechanics condition of no penetration at smaller length scales, i.e. below the resolution used in the discretisation, resulting in an infinite stiffness was used by e.g. Larsson [5, 6] . A more common method to model the tread/road contact is to use a set of uncoupled springs with constant stiffness (e.g. [7-10]). An elastic half-space formulation has also been widely used for tyre/road contact (e.g. [11 13]) but also in classical contact mechanics (e.g. [14]). The uncoupled springs neglect the coupling of displacements within the tread. The elastic half-space includes the coupling but assumes infinite wave speed, i.e. the system reacts instantaneously everywhere. Neither one of these approaches includes the effect of inertia or losses within the tread when it is deformed. They also assume that the forces at the belt-tread interface are the same as on the tread surface. Another drawback is the difficulty in finding the single-valued stiffness of the bedding springs or the elastic half-space from the frequencydependent Young's modulus and loss factor of the tread material. In practice, these parameters have instead to be tuned until correct static deformation is obtained [7, 15]. By this procedure, the softening effect of small-scale road roughness is also included in an approximative way. Losses within the tread have been included in brush spring models by e.g. Liu et al. in [16]. In [17] they show that the contact force increases with indentation speed due to the tread rubber viscoelasticity. Another recent approach to include viscoelastic effects was presented by Dubois et.al. 18]. Lopez Arteaga presented a 2D rolling resistance model in [19] where the tread dynamic was modelled using linear spring damper systems with frequency independent viscous damping. The model also included non-linear contact springs to account for the small scale roughness of the road, first described by Andersson and Kropp 
in [20]. Effects of tread inertia are included in tyre/road interaction simulations that use finite element models. Fadavi [21] calculated the forced response of a rolling tyre in contact with a flat rigid substrate. The response of loaded, rotating tyres was also modelled by Brinkmeier et al. in [22] and by Lopez Arteaga in [23]. However, finite element approaches are limited by their mesh size which affects both the frequency range of the results and the ability to capture local deformation. Sabiniarz [24], and more recently Hoever et al. [25 27] calculated the influence matrix for the contact problem from a detailed Wave Guide Finite Element model. Inertia and damping is in this way included in the description of the tread deformation. Linear contact springs were used in rolling simulations to account for the small scale roughness within an element. Hoever and Kropp [27] especially emphasised the influence of this stiffness parameter on the generated tyre/road noise. The absence of a straightforward way of deducing what the correct value of the spring stiffness should be was also stressed.

As the contact stiffness represents displacement of tread material, it will exhibit inherent mass inertia and damping characteristics. How important these dynamic features are will depend on the material properties, the spatial discretisation and the contact process itself. A discussion about the effects of tread mass and energy losses within the tread material is missing in many of the existing tyre/road contact models. As a consequence, the possible limitations due to these parameters are not fully understood.

The objective of the present work is to investigate the importance of including the tread dynamics in a tyre/road contact model. A numerical contact model is used for this purpose which simulates the detailed contact between an elastic layer and a patch of a rough road surface. The contact model was first presented in [20] where results were shown for an infinitely slow contact process with a quasi-static response of the tread. In the present paper the model is implemented on time dependent contact scenarios. Effects of tread inertia and damping are included by applying an elastic layer model similar to the one suggested by Larsson [4]. The focus in the present work is on contact forces and contact stiffness over a small road patch and how these develop during the contact. Material parameters of the elastic layer are varied to examine the effect of inertia and energy losses.

The paper is organised as follows: the next section presents the numerical contact model that is used to simulate the interaction between an elastic layer and the road surface. Section 3 presents the cases studied and the input data used in the model for the contact simulations. Section 4 presents and 
discusses the results from the simulations and the implications with respect to the modelling of the tread. Finally, the work is concluded.

\section{Contact model}

The contact model used in this investigation is based on [20] and is formulated in the time domain. The main feature of the model is the ability to account for the effect of small-scale roughness of the surfaces by local constitutive interfacial relations between the contact elements of the tread and the road. In terms of a mechanical system, these relations can be seen as non-linear contact springs with increasing spring stiffness as they get compressed. The modelling technique is in the following briefly demonstrated for multipoint contact, for a complete description the reader is referred to [20].

The contact geometry of two bodies with rough surfaces is discretised into elements on a regular grid in the numerical model. For each element on one surface there is a corresponding matching element on the other (Fig. 1). In this way pairs of contact elements are formed. The position of each element is represented by a single matching point whose height is chosen to be that of the highest peak within the element. The softening effect of the unique smallscale roughness within an element is accounted for by adding a non-linear spring between the matching points.

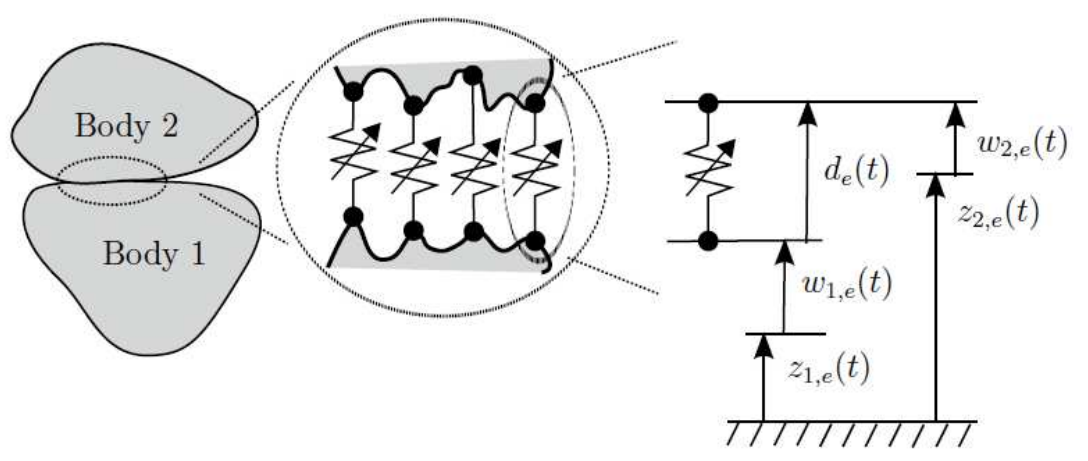

Figure 1: Geometry and modelling approach of the problem.

The distance $z_{1, e}$ gives the position of the matching point of element $e$ at the surface of body 1 when no forces act on the body, and $z_{2, e}$ is the corresponding position of the matching point on body 2 . The distance is given 
relative to a reference plane perpendicular to the direction of the contact. The distances $w_{1, e}(t)$ and $w_{2, e}(t)$ are the displacements of the matching points of body 1 and body 2, respectively, due to deformations of the bulk of the objects. The distance between two matching points is $d_{e}(t)$. Hence negative $d_{e}(t)$ means that the surfaces indent each other on smaller length-scales. The compression of the non-linear spring, $\zeta_{e}(t)$, is given by the negative separation distance

$$
\zeta_{e}(t)=-d_{e}(t)=-z_{2, e}(t)-w_{2, e}(t)+z_{1, e}(t)+w_{1, e}(t)
$$

For the case of a multipoint contact, the geometry of both surfaces is discretised in $M$ elements, numbered $1, \ldots, M$. Since the impedance of the wearing course of the road is several orders higher than the impedance of the tread, it is assumed that the road surface is rigid and does not deform

$$
w_{1, e}(t)=0 \quad \text { for } \quad e=1, \ldots, M \text {. }
$$

The displacement $w_{2, m e}$ of an element $e$ on body 2 due to the contact force $F_{m}(t)$ at any matching point $m \in\{1, \ldots, M\}$ is calculated by a convolution integral

$$
w_{2, m e}(t)=F_{m}(t) * g_{m, e}(t),
$$

where $*$ denotes the convolution operation and $g_{m, e}(t)$ denotes the displacement Green's function of the body at element $e$ due to a pressure impulse that is uniformly distributed over element $m$ and that amounts to a unitary force. The total displacement response at element $e, w_{2, e}$ is given by a summation over the contribution from all contact forces

$$
w_{2, e}(t)=\sum_{m=1}^{M} w_{2, m e}(t)=\sum_{m=1}^{M} F_{m}(t) * g_{m, e}(t) .
$$

The spring force at element $m$ is given by an integration over its stiffness function $k_{m}(x)$, which describes the interfacial spring stiffness due to smallscale surface roughness as a function of indentation

$$
F_{m}(t)=\int_{-\infty}^{\zeta_{m}(t)} k_{m}(x) \mathrm{d} x
$$

The geometrical condition at the same element $m$ is

$$
\zeta_{m}(t)=-z_{2, m}(t)-w_{2, m}(t)+z_{1, m}(t)
$$


Equations (2), (4), (5) and (6) describe a general way of incorporating nonlinear stiffness between matching points. The functions $k_{m}$ must be given in order to solve the system. Notice that all elements on body 2 are coupled via the Green function in Eq. (4), but the constitutive relation at element $m$, described by Eq. (5), is independent of the compressions of the springs at adjacent elements.

The spring stiffness function of the non-linear spring within each pair of contact elements is determined from a detailed scan of the surface geometry, the elastic modulus of the tread material, and a model of a flat circular punch indenting an elastic layer [28]. The procedure is described thoroughly in [20]. The approach yields an estimation of a stiffness function that is unique for each pair of contact elements.

To solve the multipoint contact problem, time discretisation is employed with a constant time step $\Delta t$, giving the sampling frequency $f_{s}=\Delta t^{-1}$. The force records as well as the response records are low-pass filtered and resampled versions of the continuous signals. A solution to the contact problem is found in each time step by performing a Newton-Raphson iteration.

\section{Simulations}

The previously described contact model is used to simulate the time dependent contact between an elastic layer and a rough road surface patch. A contact scenario is used where the layer is forced vertically into the road surface according to a predetermined indentation as a function of time. Dynamic calculations with a time evolution of the elastic layer impulse responses are compared with results when using a quasi-static approximation of the response. The latter only consisting of stiffness, missing any effect of material mass or damping.

Three constant indentation accelerations are used to force the elastic layer into the road surface: $50 \mathrm{~m} \mathrm{~s}^{-2}, 250 \mathrm{~m} \mathrm{~s}^{-2}$ and $500 \mathrm{~m} \mathrm{~s}^{-2}$. Notice that accelerations in the order of $1000 \mathrm{~m} \mathrm{~s}^{-2}$ are found from measurements on rolling tyres [15].

The set of Green's functions representing the impulse response of the elastic layer is obtained by a modified version of the two-layered elastic plate model presented and validated in [3, 29]. The method yields frequency response functions, which are then transformed to the time domain by an inverse Fourier transform technique. The Green's functions in the present implementation are obtained using input data representing a $1.0 \mathrm{~cm}$ thick 
elastic layer with a perfectly rigid backing. A sampling frequency of 51.2 $\mathrm{kHz}$ is used, the same as in the contact algorithm, and the resulting Green's functions are 256 samples long. Density and loss factor of the elastic layer are varied in the calculations. The default density is $1100 \mathrm{~kg} \mathrm{~m}^{-3}$. The basic setting of the store and loss modulus are shown in Fig. 2. The data is based on results provided by a tyre manufacturer and is extrapolated to higher frequencies using the results of [1]. Notice especially how the modulus increases with frequency. When varying the loss factor, the absolute value of the Young's modulus is kept constant to preserve the material stiffness. It is mainly the high frequency content of the elastic layer response that is modified when material properties are varied as can be seen in Fig. 3 where the frequency response functions at the excitation element are shown.

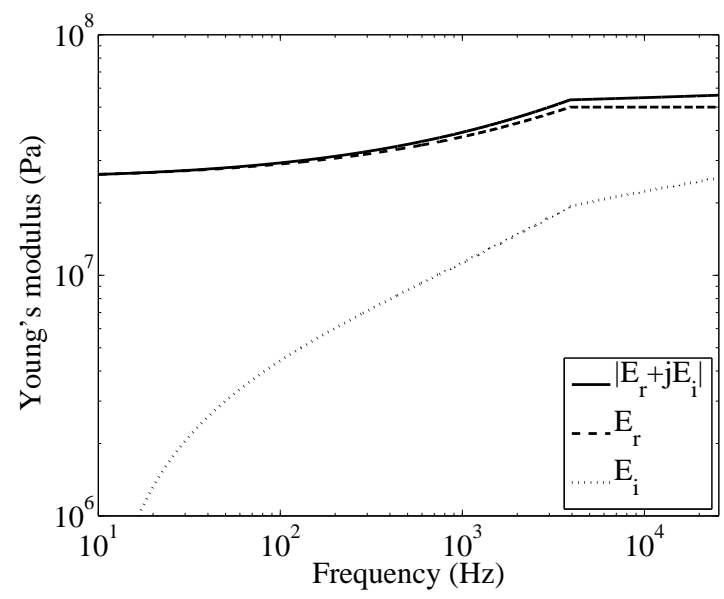

Figure 2: Young's modulus of the elastic layer.

The road surface data originates from a high resolution scan of the wearing course of a road built according to the standard ISO10844 [30]. Fig. 4 shows the road patch and indicates that a contact process will initialise at the peaks of asperities. The original resolution of the scan is $38 \mu \mathrm{m}$. This is the lower limit of what is referred to as small-scale roughness in this paper. The common assumption of a rigid road is adopted and the road data enter the contact algorithm as constants. The contact between the elastic layer and the road surface is made over an apparent area of $2 \times 2 \mathrm{~cm}^{2}$, which is divided into $20 \times 20$ elements in the contact calculations. 


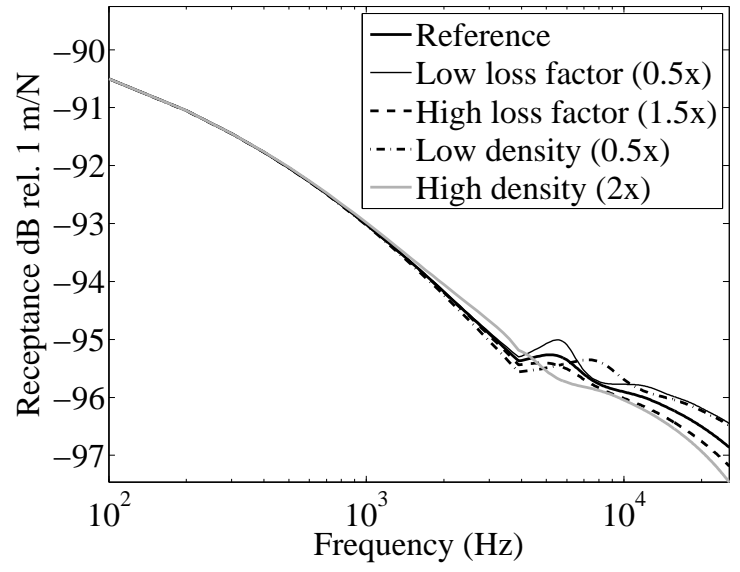

Figure 3: Frequency response of the elastic layer at the excitation element.

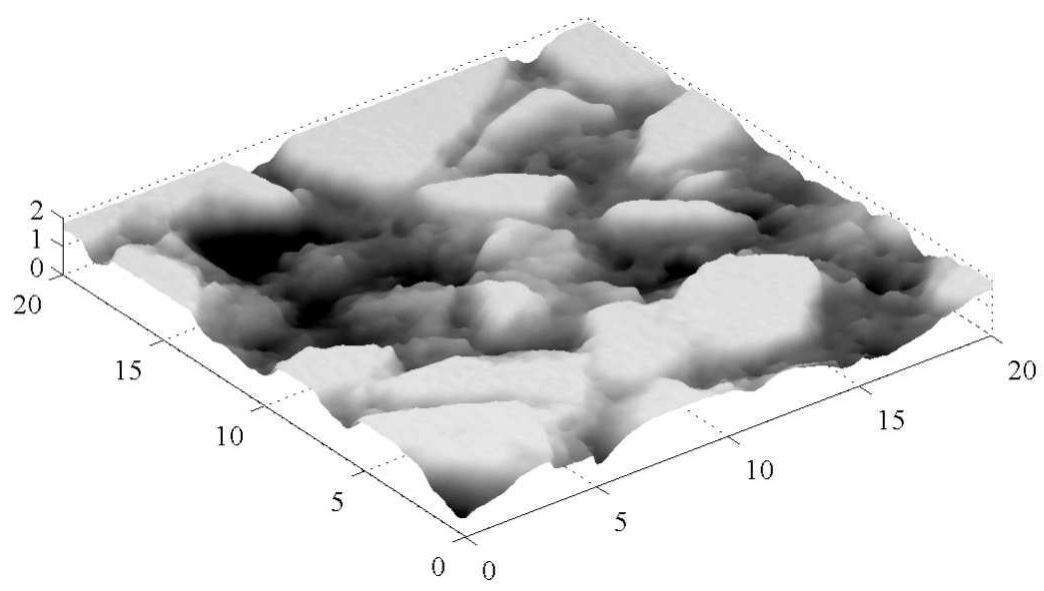

Figure 4: Road surface scan [20]. Distances in mm. 


\section{Results and Discussion}

The following section presents and discusses results in terms of total contact force in the normal direction and contact stiffness over the patch as function of indentation. The contact stiffness is defined as the derivative of the total contact force with respect to indentation.

Simulations of quasi-static and dynamic contact forces as function of indentation show comparable tendencies (Fig. 5). The contact stiffness is however substantially higher when using a dynamic response and it increases with increasing indentation acceleration (Fig. 6). The number of elements in contact grows rapidly up to an indentation of around $0.05 \mathrm{~mm}$ and the contact stiffness follows the same trend. The shape of the contact stiffness function varies in this region. For the fastest indentation it experiences a spasmodic increase whereas the quasi-static has a smooth increase. At an indentation of $0.15 \mathrm{~mm}$, about $50 \%$ of the total number of elements are in contact. Here the contact stiffness ceases to increase and it may be considered as saturated. A corresponding study of the pressure at the rigid backing gives equivalent results.

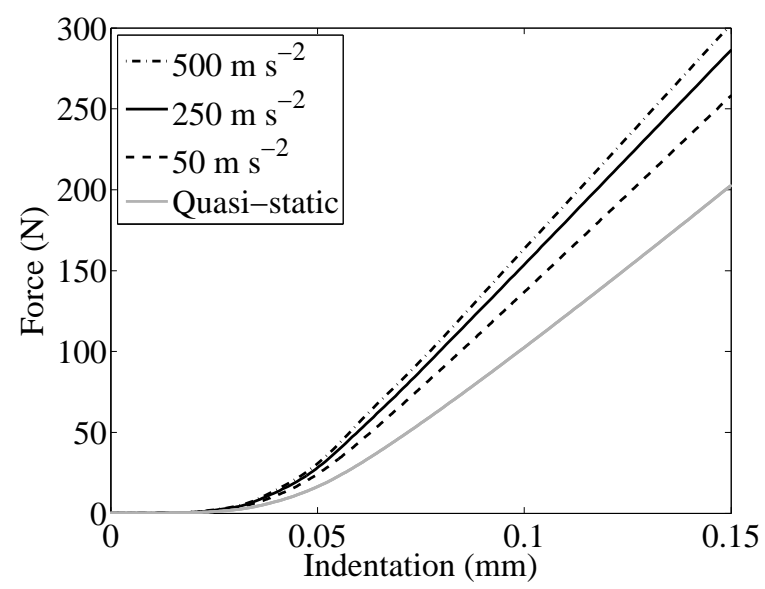

Figure 5: Contact force as function of indentation for different predetermined indentation accelerations and the quasi-static case.

The influence of damping in the material is studied by varying the loss factor of the elastic layer and comparing the results with a reference case from Fig. 6. The comparison is shown in Fig. 7 for a predetermined indentation acceleration of $500 \mathrm{~m} \mathrm{~s}^{-2}$. Increasing the loss factor gives a slightly 


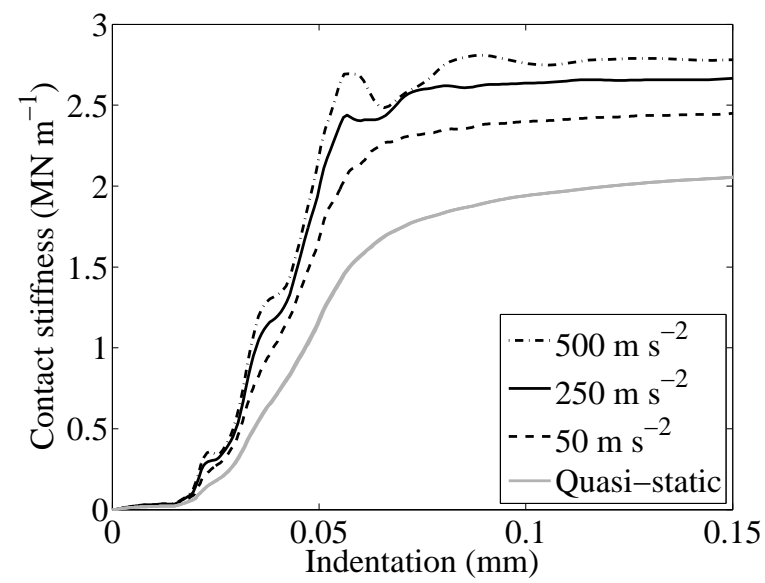

Figure 6: Contact stiffness as function of indentation for different predetermined indentation accelerations and the quasi-static case.

stiffer saturated dynamic contact and vice versa, though the character of the stiffness curve is similar to the reference case. The change in the saturated contact stiffness is around $3 \%$ for a $50 \%$ variation of the loss factor. The dynamical results can be directly connected to changes in the elastic layer Green's functions where a higher loss factor gives a smaller displacement in the first time steps.

Varying the density of the elastic layer within reason is not expected to induce major effects on the contact force. The force required to accelerate a $2 \times 2 \times 1 \mathrm{~cm}^{3}$ free, rigid segment of the elastic layer at $500 \mathrm{~m} \mathrm{~s}^{-2}$ is on the order of a few Newtons. This is a small fraction of the forces present during normal tyre/road contact conditions. Indeed, increasing the density with a factor two only affects the contact stiffness slightly, Fig. 8. Increasing the density further gives more pronounced ripples in the stiffness function. Lowering the density by a factor two also results in very small changes compared with the reference case. Especially noticeable is the fact that changes of density does not affect the contact stiffness after saturation; all cases end up with a final stiffness around $2.75 \mathrm{MN} \mathrm{m}^{-1}$. Further investigations have excluded that the ripples seen in the stiffness functions may be due to waves reflected at the rigid backing.

The results presented here imply that the shape of the stiffness function origins from a combination of the road topography and the inertia of the 


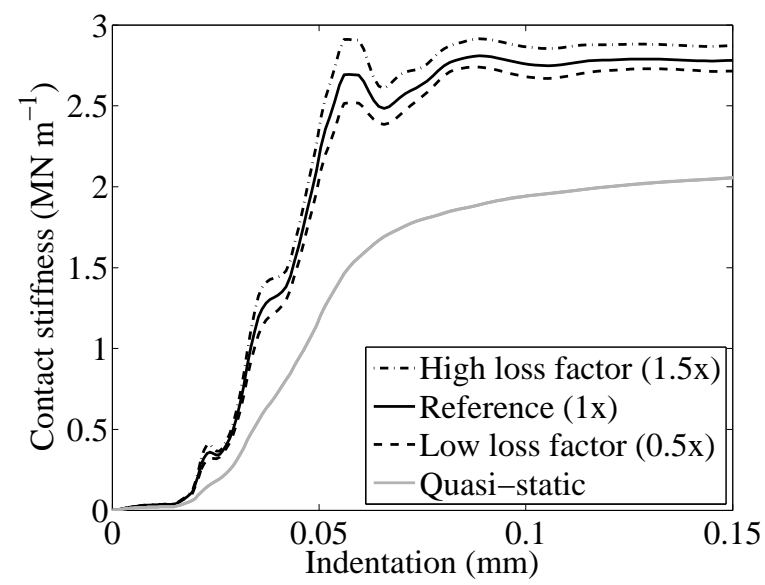

Figure 7: Contact stiffness as function of indentation when varying the loss factor of the elastic layer. A predetermined indentation acceleration of $500 \mathrm{~m} \mathrm{~s}^{-2}$ is used.

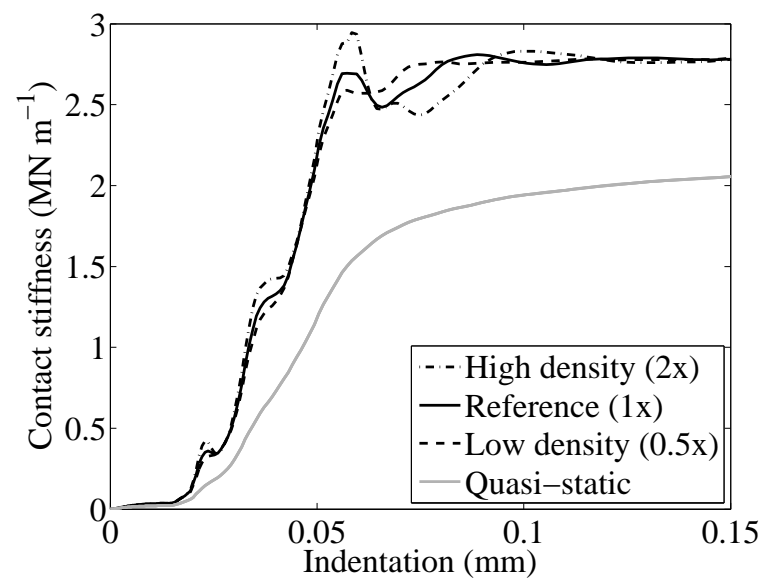

Figure 8: Contact stiffness as function of indentation when varying the density of the elastic layer. A predetermined indentation acceleration of $500 \mathrm{~m} \mathrm{~s}^{-2}$ is used. 
elastic layer material, the mass giving rise to ripples in the stiffness function. Damping in the elastic layer gives a stiffer contact compared with the quasistatic case. The effect is significant but it does not alter the character of the stiffness function. This indicates that the dynamical effects of damping could be approximated by an increased quasi-static stiffness for rougher estimations of, for instance, the contact forces in the tyre/road contact. However, as the contact stiffness also depends on the acceleration of the indentation, the stiffness must be tuned for a specific indentation acceleration. Simulations of detailed interfacial behaviour generally require the energy losses to be included in more detail, especially if rolling losses, stick-slip or stick-snap processes are studied.

When expanding the results to more complete tyre/road contact situations, a few possibly relevant limitations can be highlighted.

To focus on the tread layer of a tyre, a contact scenario with a constant predetermined indentation acceleration along with a perfectly rigid backing of the elastic layer is studied. It is a well-defined case and the accelerations used are in the order of what can be expected in real tyre/road interaction. In a real situation with a flexible belt including wave propagation and modes, the results may be slightly affected but this is not further investigated here.

To focus on the influence of the tread material properties, the response of a smooth elastic layer is used in the simulations. This situation could directly be a good representation of the tread of slick tyre, but not necessarily of a patterned tread. Finite sized tread blocks and their edge effects, i.e. softening effects close to tread block borders (e.g. Liu [17]) are not studied here.

When a real tyre is rolling on a road, the contact between a tread block and the road surface has a certain curvature. This situation is simplified to a pure vertical motion in this paper.

\section{Conclusions}

This study investigates how common approximations of the tread in tyre/road contact models may affect simulations of contact forces on a detailed level. This is done by comparing dynamic calculations that include effects of mass and damping with results when using a quasi-static approximation.

As expected, the force-indentation function is found to be non-linear for a tread, here modelled as an elastic layer, indenting into a patch of rough road surface. 
There are clear differences between simulation results when using the full dynamic impulse response of the elastic layer compared with when using a quasi-static approximation. When using a dynamic response of the elastic layer, the contact stiffness increases with increasing predetermined indentation acceleration. The contact stiffness is significantly softer, and impervious to the indentation acceleration for the case of a quasi-static response.

Any tread model that assumes a constant contact stiffness has to linearise the force-indentation curve at a specific working point to find the estimate value of the stiffness. This study has shown that the accuracy of such an approximation depends on several factors. The stiffness depends strongly on the indentation due to the growth of the contact area within the patch and within individual elements. For a specific indentation, the stiffness also depends on the initial conditions and the contact history, a hasty indentation results in significantly stiffer contact than a slow.

Damping and mass is what cause the deviations between dynamic and quasi-static results. A purely elastic, massless material would respond according to the quasi-static response. This study has shown that energy losses within the material have a significant effect on the contact stiffness. The character of the stiffness-indentation curve is, however, unaffected by changes in loss factor. This indicates that the effect of energy losses could approximately be included as an increased stiffness in a quasi-static tread model. If detailed behaviour at the tyre/road interface is to be simulated, e.g. for calculation of rolling losses in the tread and friction at the interface, it may require losses to be included more accurately. The material density does not produce any significant effects on the contact stiffness after saturation. Its major effect is small ripples on the stiffness function during the initial indentation. This indicates that the inertial effects are only of importance in very detailed modelling of the interfacial behaviour.

\section{Acknowledgements}

This work was financially supported by the Swedish Research Council (registration number 2007-4185).

\section{References}

[1] M.-J. Wang, S. X. Lu, K. Mahmud, Carbon-silica dual-phase filler, a new-generation reinforcing agent for rubber. Part VI. Time-temperature 
superposition of dynamic properties of carbon-silica-dual-phase-fillerfilled vulcanizates, Journal of Polymer Science Part B: Polymer Physics 38 (2000) 1240-1249.

[2] W. Kropp, Structure-borne sound on a smooth tyre, Applied Acoustics 26 (1989) 181-192.

[3] P. B. U. Andersson, K. Larsson, F. Wullens, W. Kropp, High frequency dynamic behaviour of smooth and patterned passenger car tyres, Acta Acustica united with Acustica 90 (2004) 445-456.

[4] K. Larsson, W. Kropp, A high-frequency three-dimensional tyre model based on two coupled elastic layers, Journal of Sound and Vibration 253 (2002) 889-908.

[5] K. Larsson, Numerical modelling of the tyre/road contact, in: Proceedings, Forum Acusticum, Sevilla, Spain, 2002, Sevilla, Spain.

[6] K. Larsson, Modelling of Dynamic Contact. Exemplified on the Tyre/Road Noise., Ph.D. thesis, Division of Applied Acoustics, Chalmers University of Technology, Göteborg, Sweden, 2002.

[7] W. Kropp, Ein Modell zur Beschreibung des Rollgeräusches eines unprofilierten Gürtelreifens auf rauher Strassenoberfläche, (A model for the description of the rolling noise from a smooth tyre on a rough road)., Ph.D. thesis, Fortschritt-Berichte Reihe 11, Nr 166, VDI Verlag, Düsseldorf, 1992.

[8] J. F. Hamet, P. H. Klein, Use of a rolling model for the study of the correlation between road texture and tire noise, in: Proceedings of Internoise 2001, The Hague, The Netherlands, pp. 1-6.

[9] E. Gerretsen, E. D. Schoen, F. J. M. van der Eerden, E. Mulder, Relevant parameters for low-noise tyre designs - and optimisation study, Technical Report TNO report HAG-RPT-010163, TNO-TPD, Sound and Vibration Division, Delft, The Netherlands, 2002.

[10] D. J. O'Boy, A. P. Dowling, Tyre/road interaction noise-numerical noise prediction of a patterned tyre on a rough road surface, Journal of Sound and Vibration 323 (2009) 270-291. 
[11] F. Wullens, W. Kropp, A three-dimensional contact model for tyre/road interaction in rolling conditions, Acta Acustica united with Acustica 90 (2004) 702-711.

[12] G. Dubois, J. Cesbron, H. P. Yin, F. Anfosso-Lédée, Numerical evaluation of tyre/road contact pressures using a multi-asperity approach, International Journal of Mechanical Sciences 54 (2012) 84-94.

[13] G. Dubois, J. Cesbron, H. P. Yin, F. Anfosso-Lédée, D. Duhamel, Statistical estimation of low frequency tyre/road noise from numerical contact forces, Applied Acoustics 74 (2013) 1085-1093.

[14] J. J. Kalker, Three-Dimensional Elastic Bodies in Rolling Contact, Solid Mechanics and Its Applications, Springer, 1990.

[15] F. Wullens, Excitation of Tyre Vibrations due o Tyre/Road Interaction, Ph.D. thesis, Division of Applied Acoustics, Chalmers University of Technology, Göteborg, Sweden, 2004.

[16] F. Liu, M. P. F. Sutcliffe, W. R. Graham, Prediction of tread block forces for a free-rolling tyre in contact with a smooth road, Wear 269 (2010) 672-683.

[17] F. Liu, M. P. F. Sutcliffe, W. R. Graham, Prediction of tread block forces for a free-rolling tyre in contact with a rough road, Wear 282-283 (2012) 1-11.

[18] G. Dubois, J. Cesbron, H. P. Yin, F. Anfosso-Ledee, Macro-scale approach for rough frictionless multi-indentation on a viscoelastic halfspace, Wear 272 (2011) 69-78.

[19] I. Lopez Arteaga, Influence of material damping on the prediction of road texture and tread pattern related rolling resistance, in: Proceedings of ISMA2010 including USD2010, pp. 4039-4052.

[20] P. B. U. Andersson, W. Kropp, Time domain contact model for tyre/road interaction including nonlinear contact stiffness due to smallscale roughness, Journal of Sound and Vibration 318 (2008) 296-312.

[21] A. Fadavi, D. Duhamel, H. P. Yin, Tyre/road noise: Finite element modeling of tyre vibrations, in: Proceedings of Internoise 2001, The Hague, The Netherlands. 
[22] M. Brinkmeier, U. Nackenhorst, S. Petersen, O. von Estorff, A finite element approach for the simulation of tire rolling noise, Journal of Sound and Vibration 309 (2008) 20-39.

[23] I. Lopez Arteaga, Green's functions for a loaded rolling tyre, International Journal of Solids and Structures 48 (2011) 3462-3470.

[24] P. Sabiniarz, Modelling the vibrations on a rolling tyre and their relation to exterior and interior noise, Ph.D. thesis, Division of Applied Acoustics, Chalmers University of Technology, Göteborg, 2011.

[25] C. Hoever, P. Sabiniarz, W. Kropp, Waveguide-finite-element based parameter study of car tyre rolling losses, in: Proceedings - 6th Forum Acusticum 2011, Aalborg, Denmark, pp. 789-794.

[26] C. Hoever, W. Kropp, A simulation-based parameter study of car tyre rolling losses and sound generation, in: Proceedings - 9th European Conference on Noise Control, EURONOISE 2012, Prague, Czech Republic, pp. 926-931.

[27] C. Hoever, W. Kropp, The influence of modelling parameters on the simulation of car tyre rolling noise and rolling resistance, in: Proceedings of DAGA-AIA 2013, Merano, Italy pp. 1601-1604.

[28] F. Yang, Indentation of an incompressible elastic film, Mechanics of Materials 30 (1998) 275-286.

[29] K. Larsson, S. Barrelet, W. Kropp, The modelling of the dynamic behaviour of tyre tread blocks, Applied Acoustics 63 (2002) 659-677.

[30] ISO 10844, Acoustics - Specification of Test Tracks for the Purpose of Measuring Noise Emitted by Road Vehicles, International Organization for Standardization, 1994. 